\title{
Study on Smart Clothing Application Based on Interaction Technique Concept
}

\author{
Jinqiang Li \\ Shandong Vocational Institute of Clothing Technology, Tai'an, 271000, China
}

Keywords: Interaction technique, Smart clothing, Concept, Experience, Interaction

\begin{abstract}
Interaction design is a frontier concept of era development, and it can realize the perfect coexistence of sensibility and rationality and satisfy people's curiosity about new things and high-grade emotional experience. Nowadays, to fit the society and people's demand for high-quality life, clothing has firstly integrated the intelligent design and then added the interaction concept, which is called "intelligent interaction design of clothing design" in the industry. This paper briefly introduces the basic thought of interaction technique and shares the development and application of some interactive smart clothing products in the context of modern science and technology era.
\end{abstract}

\section{Introduction}

Besides paying attention to intelligent functions, the current product design has also integrated more interaction design that can trigger people's nerve and emotion. The smart clothing based on interaction technique concept starts from paying attention to people's psychological and behavioral actions and emphasizes the interactive relationship between human and human, between human and things, and between human and environment, so as to reach a high level of emotional and spiritual satisfaction.

\section{Interaction design}

\section{Connotation of interaction design}

Interaction design is a kind of external element design that can link together the product, environment and system for interaction and pass such interaction out. In brief, interaction design can express, describe and show out the function of interaction between the product and other factors. In the current context of extensive development of the man-machine interaction system, the interaction design concept allows the existence of more possibilities and more combination patterns, and has quietly become an integral part of people's life.

\section{Though of interaction design}

Interaction design derives from the "man-machine interaction" concept, and has gradually been closely linked to people's life. The designer Gurdin.J once said, at the annual ACM CHI'90 meeting held in Seattle of the U.S., that the interaction design had moved from the field of machinery towards the society and people's life and had continued the vitality of computer development. While, the famous American rhetorician Kenneth Burke tactfully defined the theory of interaction design, in his A Grammar of Motives, as "an attitude or action formed when a group of people apply the language to another group of people". In our country, a magazine named Design mentioned the six elements of interaction design: human, tool, action, medium, motive and scene, which exactly fitted in with the perception of behavior attributes in sociology. In the eyes of modern people, the traditional logical thought and design concept have fallen behind, and the products with interaction design that can redefine the material, color, structure and function of things and even the thought have redefined the thought of the society and provided a new environment for people's spiritual and material experience.

\section{Essence and purpose of interaction design}

Interaction design pays attention to not only the pleasant form design, but also the effective action organization and strict logical structure; these are what can really solve the practical problems. In other words, interaction design is an action used applied by people to express the communication 
between human and product, and it contains people's expectations of the product; this is also what the interaction design really expresses in essence.

Since the essence of interaction design is to express the communication between human and things, its purpose is to facilitate people's obtaining better experience. The mechanism of interaction between artifacts and human can exactly improve the user's experience level and purpose. It is undoubtedly a clever design to guide people's interaction with the product that contains the interaction technique concept. Therefore, interaction design should be a kind of mechanism, which should be established on the basis of considering people's experience background, application experience and emotional amalgamation and finally fitting them in the user's thinking logic to become an integral part of human.

\section{Interaction design in clothing product}

Nowadays, interaction design exists in people's various production and living fields. The clothing design has also introduced a lot of interaction design concept; what the designer concerns is the user's psychological behaviors and consumption features, and what the designer considers is how to realize the bidirectional interaction between the user, as the core of the interaction, and the clothing.

\section{Smart clothing and interaction design}

In brief, product intellectualization is to give vitality to products. In the field of clothing design, the smart clothing with life system simulation is more widely accepted by people. Just like human beings themselves, the smart clothing can really have the perceptivity and reaction to all the stimulations, thus it is a product of the new era where electronic technology is incorporated in textile materials. For modern science and technology, intellectualization reflects humanity more or less; this is the mechanism that the smart clothing really expresses; it will make reactions accordingly at any time by perceiving the changes of external environment and internal state, thus, people call it the fuzzy perceptual characteristics and concrete feedback expression. Besides, people can even add some whimsical ideas into the design of smart clothing; it is such design demand for smart clothing that makes people think about the connection between interaction design and smart clothing.

\section{Preconditions for integrating smart clothing with interaction concept}

Interaction technique is a sensing technology and is a kind of important motivation for people to pursue high-quality experience. That is to say, people hope that the objects they used can react to their demands, and even think about what they want and meet their desire for control. Therefore, the integration of interaction design and smart clothing becomes inevitable for development. At present, there are many preconditions for integrating designed smart clothing with interaction technique concept; for instance, clothing shall be designed in a way that its usability is guaranteed. The usability includes two aspects: on one hand, it refers to the comfort usability based on traditional clothing, namely the practicability; clothing shall be designed to meet the aesthetic feeling, modern people's personalized needs and the most basic comfort; on the other hand, it refers to the usability of intelligent system, that is to say, the smart technology theme that the smart clothing hopes to express can be brought into play when the clothing is used, such as the light, thin and sweat-absorbent jersey designed by Adidas for sports. Such jersey will not steal the show during the sportsman's exercise, and its light and thin material is more conducive to the sportsman's exercise just like that the sportsman does not wear it. Besides, it shall have strong air permeability and sweat-absorbing ability; this is the smart clothing's intelligent sensing feedback for people, which keeps the sportsman's body dry during the whole process of exercise and thereby guarantee certain athletic ability of the sportsman. Moreover, just like the ordinary clothing material, the material of the jersey shall be cleanable and can be used repeatedly, and its security shall be taken into consideration during design.

\section{Functional design of smart clothing based on interaction concept}

For human beings, the earliest clothing with interaction technique concept should be the space suit worn on the spaceman who was sent into space; it was really applied with extremely complex space technology and intelligent system. With gradual humanization of the era and technology, the smart clothing with abundant interaction technique concept gradually comes down from the altar of high 
technology and slowly blends in design and application of folk clothing. This is dominated by the inevitable tendency and needs of ceaseless market space expansion and commercialized development. Smart clothing can change people's living habits, while interaction technique adds more considerate humanized design and application functions; the commercial value of such clothing design will inevitably increase. Therefore, intelligent functions and interaction technique concept of smarting clothing can be integrated and realized by the following means.

\section{Application of smart fiber}

To make clothing become "smart" and humanized, smart fiber shall be applied. At present, smart clothing is basically is made based on the thought of interlacing smart fiber and ordinary fiber. Smart fiber is also called functional fiber, which involves many scientific fields, such as microelectronics, artificial intelligence, material science, neural network, and even the somatology and sports science. Integrating the technological elements of these fields is to re-endow ordinary textile materials with vitality, namely, to improve the systematicness and of textile materials and endow the macromolecular chain structure of fiber with intelligence, such as the "sharkskin swimming suit" that shined brilliantly during the Athens Olympics in 2000. This technique was a hot controversial topic in this field in that very year; it was made by applying the theories of "pressure drag", "wave drag" and "frictional drag" involved in physics, simulating the real structure of sharkskin and utilizing the non-hydrophilic Teflon fiber. After careful observation, it can be found that the surface of such swimming suit has millions of small ratchets; when water molecules flow by these ratchets, innumerable miniature eddies will be generated to keep the separation points of the suit's boundary layers at both sides of the sportsman's body ceaselessly retrograding, so as to delay and weaken the formation of eddies, make the sportsman swim easier and faster, and reduce the great pressure drag by increasing a very little frictional force. Its principle is the same as that of the golf ball with numerous small pits; the golf ball with smooth surface can be stroked out for only dozens of meters, while the golf ball with over 500 small pits can be stroked out for more than $200 \mathrm{~m}$. Although the intelligent swimming suit with high technology can bring the sportsman with only one tenth or even less advantage, the very little advantage is enough to decide the sportsman's destiny with regard to a high-level competition.

\section{Fiber modification}

Smart fiber is generated by modifying the ordinary fiber. The "Olympic dragon dress" is fabricated based on the principle of ordinary fiber regeneration. The Olympic dragon dress is accomplished with regenerated fiber that is covered with hydrophilic nonporous PU coating and applied with lamination technology. Thus, an invisible film is formed on the fabric of the dress; it has not only certain degree of waterproof capacity to avoid rainwater penetration, but also a considerable air permeability to quickly emit the internal moisture and heat through the fabric and thereby guarantee the smooth air flow between human body and dress and realize the timely adjustment.

\section{Interactive elements on smart clothing}

Smart clothing combines external interactive elements and hi-tech sensors, which is the final combining form of smart clothing and interaction design. NTT Docomo, the Japanese mobile operator, and the material giant TORAY has jointly developed a smart clothing that can detect human body's blood pressure and heart rate. It adopts the Hitoe nano-fiber, utilizes the nano-fiber coating, the sensing material developed by TORAY, and detects human body's heart rate and blood pressure using the patch attached to fiber. While, NTT Docomo provides the seamless transmission to send the data to the health application in mobile phone. Such clothing is also characterized by handiness, softness, good elasticity, etc. On this type of clothing, the patch can perfectly reflect the information data, of which the main reason is the polyester nano-fiber of 700 nanometers in diameter adopted for the clothing. These fibers have numerous voids, which are filled with conductive molecules; they can jointly form the continuous layer of resin, to facilitate the close contact between material and human skin and thereby quickly and sensitively detect various physiological data of human body. 


\section{Example of smart clothing designed with interaction concept}

On the stage, some performance costumes are applied with luminous coatings that make these costumes more brilliant under the lights and bring the audiences with a visually striking image. These are the smart performance costumes designed on the basis of interaction technique concept.

\section{Fabric selection}

Neoprene is the main garment fabric. Neoprene is the polymer of chloroprene and copolymer emulsion; it is also called diving fabric. Such fabric has extremely strong oxidative resistance and self-extinguishing resistance; its oil resistance is second only to nitrile rubber and has very good tensile strength and elasticity. Besides, it adapts to a wide range of temperature and can be used within the temperature range of $-35^{\circ} \mathrm{C} \sim 130^{\circ} \mathrm{C}$.

In the post process of clothing production, the electric leakage of clothing can be avoided with the additional circuit and light emitting diode, and such three-dimensional design also creates a body-shaping beauty.

\section{Design of sound-controlled circuit}

The composition and adjustment of sound-controlled flasher circuit system for stage performance costumes adopt the sound-to-electricity conversion mode of sound-controlled flasher circuit, and form the binary counter triggering circuit relying on the mutual coordination of amplifier and dual-D trigger CD. See Figure 1.

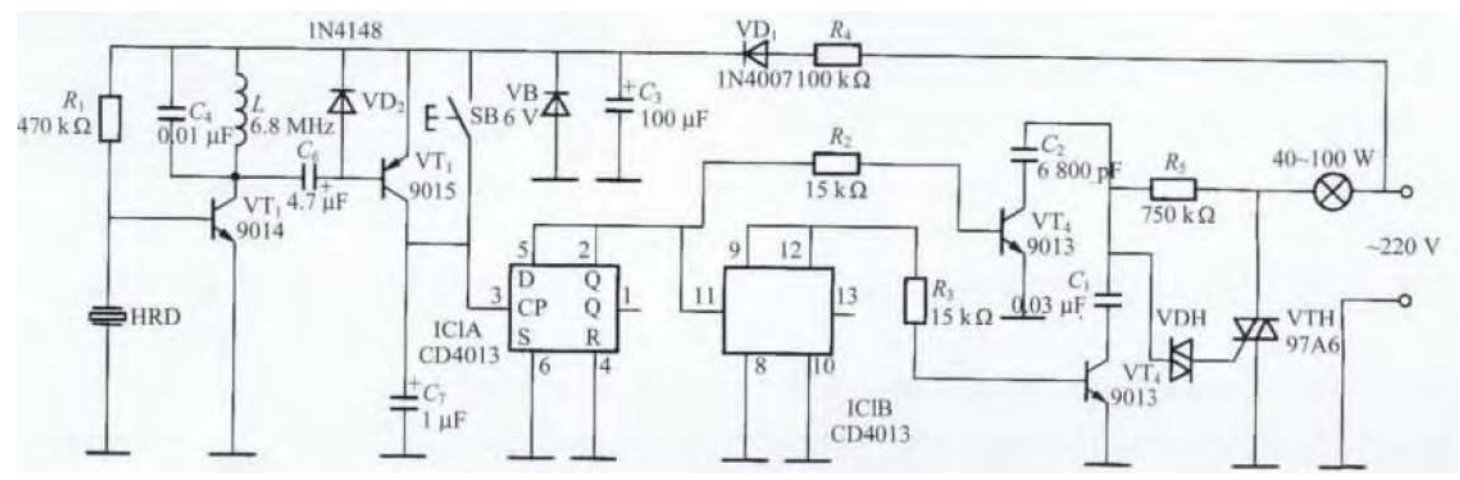

Figure 1: Design Plan of Sound-controlled Flasher Circuit

As shown in the Figure, the sound controlled system gives out sound wave of frequency by means of power supply and sends it into the electric ceramic chip HTD, and then the frequency-selective amplifier composed of $\mathrm{VT}_{1}$ elements carries out frequency amplification. The amplified signal is then detected by $\mathrm{VD}_{2}$ to realize the effect of conducting $\mathrm{VT}_{2}$. The effect is that the collector outputs the high electric level, and the high electric level signal is sent to the dual-D trigger CD; at this time, the binary counter carries out the signal counting accordingly, achieves the cycle control of $\mathrm{VT}_{3}$ and $\mathrm{VT}_{4}$ and realizes the conduction between them.

After the above-mentioned physical circuit conversion process, the glowing effect of clothing will be more beautiful than ordinary LED light, and such design can save economic cost and time cost and thereby improve the efficiency of clothing production. With the sound-controlled and optically-controlled effect, the sensing system of clothing can control the flickering of LED light bar and make the effect of clothing changed according to the stage conditions such as lighting intensity, decibel, etc. The intelligent sound-controlled flickering stage performance costumes researched and designed have a very good effect for some vocal concerts held at night. For some people who get used to riding bicycles at night, such costumes also have the functions of warning and protection to guarantee safe riding.

\section{Summary}

In general, smart clothing based on interaction technique concept plays a great role in practical application. It crosses over three fields: information science, costume design and interaction design, achieves the human-to-human and human-to-things sensing, feedback and reflection, realizes 
feedback, projection, visibility and consistency of functions. It is also inspiring for the development of future costume technology. It is the interaction technique that links people closely together with clothing, and makes people feel both physiologically and psychologically coordinated and pleasant. We can say that the smart clothing integrated with interaction technique concept is a great people-oriented invention in modern society.

\section{Acknowledgments}

This paper is the development plan subject of Taian Municipal Science and Technology Bureau: Taian Clothing Engineering Research Center 20091003, and the development plan subject of Taian Municipal Science and Technology Bureau: Construction of Clothing Technology Service System for Clothing Enterprises in Taian 20084006.

\section{References}

[1] Chen Yifan, Zhu Ruijuan, Cai Tingting, etc. Application Design of Smart Clothing Based on Interaction Technique Concept. Journal of Shanghai University of Engineering Science, 2014, $28(2)$.

[2] Meng Lei and Liu Yuan. Research on Design of Interaction Technique Based Smart Clothing for Preschoolers. Hundred Schools in Arts, 2011(5).

[3] Liu Yibo and Wang Lei. Brief Analysis on Connotation and Principle of Interaction Design of Clothing Products. Art and Design, 2014 (9). 